\section{Collective Mapping to Support Citizen-Government Interactions Using a Digital Platform}

Gl_Forum 2016, Vol.2

Page: 147-156

Full Paper

Corresponding Author:

rtapia@centrogeo.org.mx

DOI: 10.1553/giscience2016_02_s147

\author{
Rodrigo Tapia-McClung \\ Centro de Investigación en Geografía y Geomática, Mexico
}

\begin{abstract}
This paper presents a case study of user-generated geographic information in a small neighbourhood in Mexico City. It deals with citizen empowerment and actions directed to improve their local surroundings. It is constructed in a bottom-up fashion: from the citizens towards the local authorities. The exercise is carried out using an online platform developed for this purpose. The tools, methodology and lessons learned so far are described. This is an ongoing project and preliminary results are discussed. The acquisition of enough citizen data helps citizens focus their efforts when negotiating with the authorities, in situations and locations that have been found to be problematic. A digital map constructed by the citizenry is a helpful tool to locate areas of opportunity that require attention. Additionally, it is an effective communication tool to convey messages to the authorities. The identification of these types of locations is helpful in gaining insights into what, from the citizens' perspective, can cause significant discrepancies between what they observe and what is officially reported. The proposed platform incorporates a way to validate official data, a voting strategy to assess the credibility of citizen contributions, and crowdsourced information on parcel data.
\end{abstract}

\title{
Keywords:
}

volunteered geographic information; citizen science; crowdsourcing; citizen empowerment; collective mapping

\section{Indroduction}

Collective mapping can be part of crowdsourcing activities such as "generating a map using informal social networks and web 2.0 technology" (Heipke, 2010, p. 550) or the acquisition of volunteered geographic information (VGI), defined by Goodchild as "the widespread engagement of large numbers of private citizens, often with little in the way of formal qualifications, in the creation of geographic information" (2007, p. 212).

It is easy for people to be involved in mapping processes, thus becoming citizen mappers. It is increasingly common for people without qualifications in the field of Cartography to be able to contribute to local cartographic records. Citizen participation, public input and the report of social incidents have become useful avenues for interactions between citizens and authorities. When citizens provide data to the authorities, the authorities can look at things 
from a different perspective and realize different courses of action to help improve the citizens' quality of life. Citizens no longer wait for official data to be released. They become data generators. This paradigm shift fosters the existence of different mechanisms to study societal issues.

Citizen-government interactions can be broadly classified as bottom-up and top-down. Bottom-up refers to "actions conceptualized, incepted, developed and led by members of the local community" (Jovchelovitch \& Priego-Hernandez, 2015). On the other hand, top-down processes focus on the provision of these processes by the authorities for the building blocks of society. Johnson and Sieber (2013) call these "citizen-to-government" (C2G), and "government-to-citizen" (G2C), respectively.

In this research, a case study of a $C 2 \mathrm{G}$ process that focuses on the citizenry challenging the availability of official data is presented, through the use of a digital platform suitable for VGI or crowdsourcing.

One of the objectives of this research is to delve deeper into the understanding of citizen participation and the appropriation of technological aids to tackle citizen issues and concerns regarding their environment through the use of crowdsourcing and volunteered geographic information. Another goal is to provide citizens with means to become empowered. As things stand, in Mexico the only two forms of direct citizen participation are referendums and public consultations. These, nevertheless, follow the same workflow as official voting exercises emanating from the government. Efforts to provide citizens with means to express their perceptions and to be able collectively to build from them thus become relevant, as do efforts to find themselves in a position to support sound participative proposals and to articulate collaborations with their local authorities.

Technology is not an essential element, however; it is just an aid. Problems are not bound to be solved because of the existence of a new platform. Conversely, the use of pre-existing elaborate participative platforms requires a solid back-end group to guarantee that such platforms are kept functional. It is rarely the case that citizens can initiate projects with such a structure, either financially or in terms of the human resources required for it to run smoothly.

Although other platforms have already been developed for purposes similar to the one presented here, two main issues are recognized: first, citizens do not necessarily appropriate the platforms, so a lot of effort may go into the development and implementation, and no tangible results come out of it; second, during initial tests with citizens working on volunteer data collection, only basic features are commonly required by them. However, most platforms offer a wealth of functionality that typically requires a dedicated team of managers, designers, data administrators and curators. Additionally, adhering to platforms that have already been developed usually means working with what is available, the need to develop specific functionality, and a learning curve in order to get acquainted with the platform. This research paper presents advances in the design, implementation and use of a basic geospatial platform that enables citizens to collect information and use it to support citizengovernment interactions by means of a citizen agenda for use in negotiating improvements in the quality of life in a neighbourhood. 
In the remainder of this paper, a short review of other platforms is presented, followed by the case study, a brief discussion, and ideas for future work.

\section{A sample of platforms for collective mapping}

There have been a wide range of motivations, interests and applications for citizen observation and reporting platforms. The common denominator is, however, the idea that many contributors can share knowledge and observations to achieve a common goal.

Citizens can collect data on very different topics. iNaturalist lets users record observations of the natural world (California Academy of Sciences, 2015). Open'TreeMap aids in the creation of tree inventories for ecosystem management and urban forestry (2015). Ushahidi is well known for being useful in crisis management mapping (2015). GeoCitizen was developed as a platform for community-based spatial planning (Atzmanstorfer et al., 2014). GeoKey allows the creation of customized projects for citizen data collection (University College London, 2015). Most of these platforms provide both web and mobile apps. GeoKey can be configured to use the EpiCollect+ mobile app to collect data (Imperial College London, 2015). FixMyStreet is a platform that focuses on user reports about local neighbourhood problems (mySociety, 2012). Although it requires a dedicated group of administrators, designers, developers and data curators, the advantage of FixMyStreet is that it acts as a link between citizens and authorities, who can then address the problem reported. For the case presented here, unfortunately, such a relationship with local government is non-existent, although one of the goals of the project is to be able to build such a collaboration with local authorities.

One disadvantage for our purpose is that most of these platforms, except GeoKey, which is able to handle different geometries, focus on point data. With the exception of GeoCitizen, all are open source, thus making it possible to implement new functionality and extend their capabilities. One drawback, however, is that most are in English, and it is a cumbersome process to localize all front-ends for non-English speakers (Ushahidi already provides some translations). As will be explained further in Section 4, the proposed platform includes two relevant enhancements that were not found on the platforms reviewed: 1) they allow the exploration of how citizens feel about other users' contributions as a means to assess the quality, accuracy and credibility of both VGI and users; 2) they allow users to crowdsource data on parcel lots by using polygon geometries from cadastral records.

\section{The Citizen to Government (C2G) case study: the Lindavista neighbourhood}

As already stated, the dynamic of bottom-up processes is from the citizenry towards the authorities. In this case, the neighbourhood's needs are a driving force towards interaction with the authorities. Collaborating with the neighbourhood offers an opportunity to reflect on many aspects of citizen-government relations, along with how science and technology can aid in communicating citizen issues and preferences. 
The case study presented here is centred in the Lindavista neighbourhood, located north of Mexico City. It covers an area of roughly $2.1 \mathrm{~km} 2$ and has a population of approximately 15,000 in about 122 city blocks. It is a neighbourhood with a long tradition, founded in the 1930s as a result of the industrialization that was taking place. It is home to restaurants, cinemas, private schools, different types of local businesses, subway stations, malls, hospitals and two important public schools. The area used to be one of milk-producing ranches but it quickly rose to be one of the high-class neighbourhoods of the city. An important film studio was established there and inspired many artists to live in or around the neighbourhood. Writers and public figures followed suit. From the second half of the 20th century, and as a consequence of the demographic explosion, the neighbourhood suffered from overpopulation and overcrowding. After having been considered high-class, it quickly became surrounded by lower-class families, which started exerting pressure on it. Around the mid-1990s, it had become a target of mixed crime such as public assault, burglaries and kidnapping.

Neighbours complain about different aspects of their everyday life in the area, and some groups had already been working with paper maps in order to keep track of important issues spatially. They had a solid idea of the types of problems that they could observe and help resolve in cooperation with their local authorities. Throughout a series of meetings, it was agreed that collaboration could be put in place to develop a basic digital spatial platform to help them collect data. These meetings served to clarify the types of variables they were interested in observing. Given their previous work detecting problems and their focus on particular issues, it was fairly easy to define a list of categories to work on (see Table 1). In order to help citizens in data collection, care was taken so that the selected variables would be as objective as possible. As citizens participate in the problem definition, data collection and analysis, this is an extreme citizen science exercise, according to the typology proposed by Haklay (2013).

Table 1: Five categories and sub-categories for data collection

\begin{tabular}{l|l|l|l|l} 
Businesses & Schools & Security & Mobility & Cadastral \\
\hline Restaurant & Elementary school & Dark place & Pothole & Parcel lots \\
Bar & Middle school & Nonworking streetlight & Missing manhole & (levels of \\
Fixed street vendor & High school & Visibility obstacle & cover & construction) \\
Non-fixed street & University & & & \\
vendor & Combined & & &
\end{tabular}

Taking into account the strengths and limitations of the platforms reviewed, and considering there were restrictions on both personnel and budget, it was decided to implement just a basic platform that would allow citizens to crowdsource perceptions from around the neighbourhood. Once citizens had specific needs, these needs drove the development of additional functionality inside the platform. First, a simple online map was made available for users to pinpoint locations where observations on any of the categories from Table 1 were detected. It was deemed appropriate to include available data about the businesses and schools categories from official records in order to improve field data collection times. The research question concerning the credibility and accuracy of VGI is open for debate, and 
some guidelines have been put forward to assess volunteered contributions (Fonte et al., 2015). A first approach to obtain an empirical measure of the credibility and accuracy of these contributions is to use user voting. Letting users express themselves on what others have reported opens the possibility for a tacit discussion about the credibility and the validity of what is on the platform, thus allowing the measurement of accuracy by users themselves. Lastly, the proposed platform includes a way to crowdsource data on parcel polygon data.

\section{Digital platform for Lindavista}

An exploration of some of the existing platforms made it clear that focus has been on point data for citizen observations. While this is advantageous for pinpointing perceptions, some perceptions relate to larger spaces, without a defined latitude/longitude pair. As mentioned earlier, it is difficult to deploy a full participatory platform without the existence of a dedicated team behind it. For this case study, citizens needed only basic functionality, and it was deemed appropriate to develop a digital platform that suited their needs. The platform was developed using open-source software and implemented as a web app, functional in both mobile and desktop environments. The front-end uses Leaflet JS, Bootstrap and jQuery. The back-end uses PostgreSQL/PostGIS and PHP. Although digital platforms for citizen contributions are not new, the relevance of the one presented here is threefold: as a way to validate official data, as a voting strategy to assess the credibility of citizen contributions, and in allowing crowdsourcing information on parcel data, all of which are detailed below. The platform consists of two parts: the "categories" map and the "parcel lot" map.

\section{Categories map}

The categories map is an online map in which users can report observations. Local residents are in charge of data collection and quality assurance. In order to avoid fake data as much as possible, the system is designed so that only authenticated users can enter data, while visitors can navigate, explore and see collected data points. After clicking on the desired location on the map, data is entered by filling in a survey form. Information from the spatial database is displayed on the map with a clustering strategy for each category, dynamically changing when zooming in or out. This helps prevent clutter and is a visual aid. It also makes data display much more efficient in terms of platform performance, avoiding lags when zooming or panning. Collected data is also automatically inserted into a cartoDB (2015) visualization to display an animated map, useful in keeping track of how data collection is progressing over the study area.

The online map was first populated with data from a nationwide survey of economic units, available from the National Institute of Statistics and Geography (INEGI (Instituto Nacional de Estadística y Geografía), 2015). In terms of system design, this meant dealing with two separate tables in the database (one to hold the official records and initially populate the map, and the other to store citizen observations), giving citizens the opportunity to validate data from official sources (a functionality not seen in other platforms), and filtering data from the whole country to include only, for the neighbourhood under study, points in either 
the Businesses or the Schools categories. Data points added to the map had their associated information available for display on click. The system is designed so that authenticated users have additional functionality over visiting ones. When an authenticated user clicks a marker, if the official category matches one under consideration, it can be validated. If data is incorrect, the user can edit and update the information. If the marker is not relevant for the categories under scrutiny (for instance, a karate school), the user can delete it. Whenever the user takes any of these actions, the system triggers the deletion of the corresponding record in the economic units table; depending on whether a data point was edited or validated, it also triggers the insertion of a new record in the citizen observations table. Figure 1a shows an example of the validation, editing or deletion of data points.

A voting approach is used to capture users' perceptions of observations that have already been reported and allow them to express their (dis)agreement. Only authenticated users have access to this functionality; otherwise, it would be possible for anyone to vote many times for or against a particular observation. Once a user has voted, they can only change their opinion, not vote again. This is to prevent users from repeatedly voting for or against a single observation, and to try to capture the general feelings of a larger group of users. Figure 1b shows an example of the voting strategy. This is an initial approach to accommodate the issue of assessing the credibility of volunteered geographical information (Fonte et al., 2015). How can the reliability of citizen contributions be measured? Here, the idea is to quantify how many people (dis)agree with a specific report and obtain an empirical degree of trustworthiness of the observations.
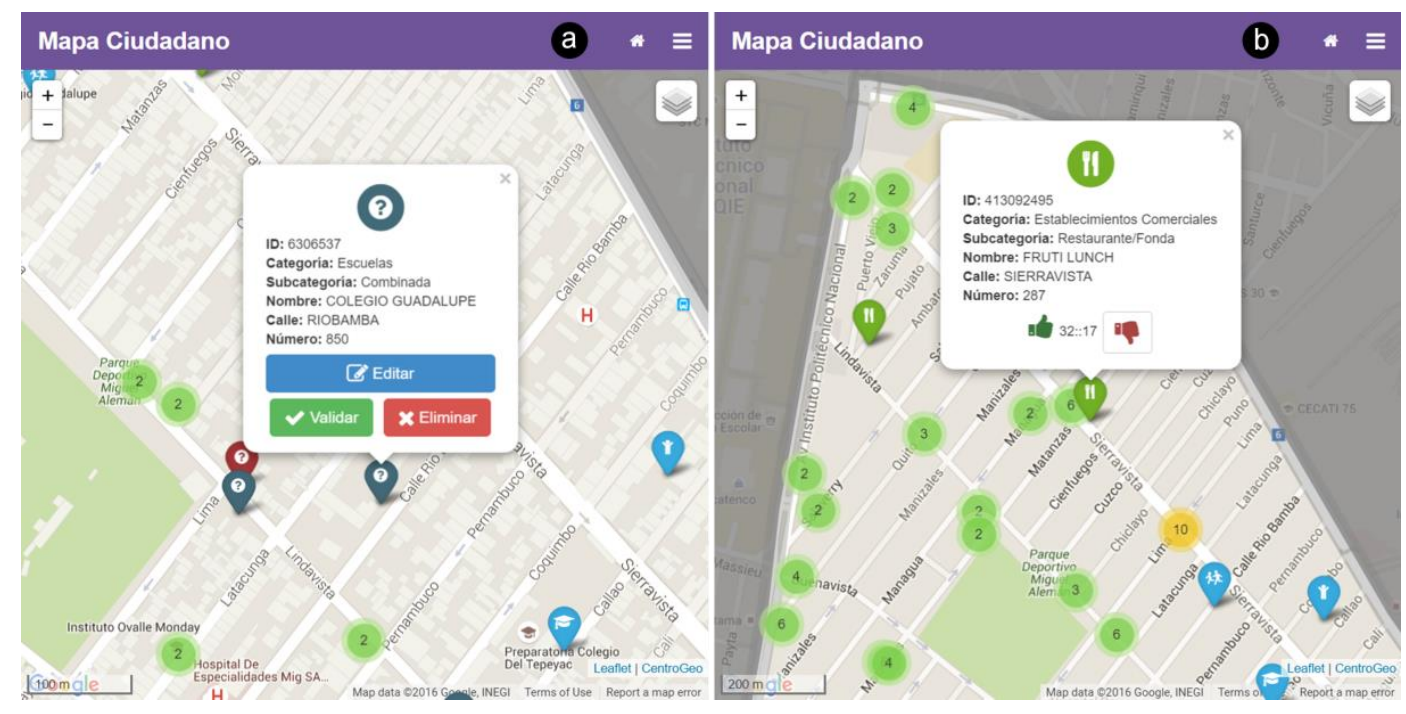

Figure 1: a) Validation, editing or deletion of data points; b) Voting strategy implemented in the platform 


\section{Parcel lot map}

Citizens in this neighbourhood are also interested in obtaining information about deviations in land use with respect to the current land use policy for the city, particularly the maximum levels of construction allowed in each parcel lot. In accordance with local law, it is possible to access public records that contain relevant information, but it is a cumbersome and sometimes lengthy process, and the data obtained may not be readily available in open formats.

Parcel geometries were obtained and stored in PostGIS. The front-end for this map is designed so that the browser queries the database, which returns a JSON feature collection containing the geometry of parcel lots along with their associated information. These are then added as a Leaflet layer on the map. The attribute for the parcels' level of construction was initialized with a null value. Parcel lots are categorized according to a coloured legend shown on the map. Clicking on a polygon displays a pop-up window with the lot id, cadastral account and current level of construction. The pop-up window is shaded with the colour corresponding to the level of construction and, again, only authenticated users can edit the information. Both coloured schemes help users get a better idea of the data. Additionally, the coloured map gives a quick overview of the spatial distribution of peaks and troughs of construction levels. Figure 2 shows an example of the spatial distribution of parcel lots with different levels of construction in the study area.

This case study is relevant as it helps citizens collect data concerning land use. This, as far as I know, has not been done in the city before. Upon completion, it will be very useful to compare citizens' observations with official figures. Initial approaches to local authorities to obtain the official land use map have not been very successful. However, the citizens' drive to obtain and make this information publicly available may set an example for the authorities to move towards open data.

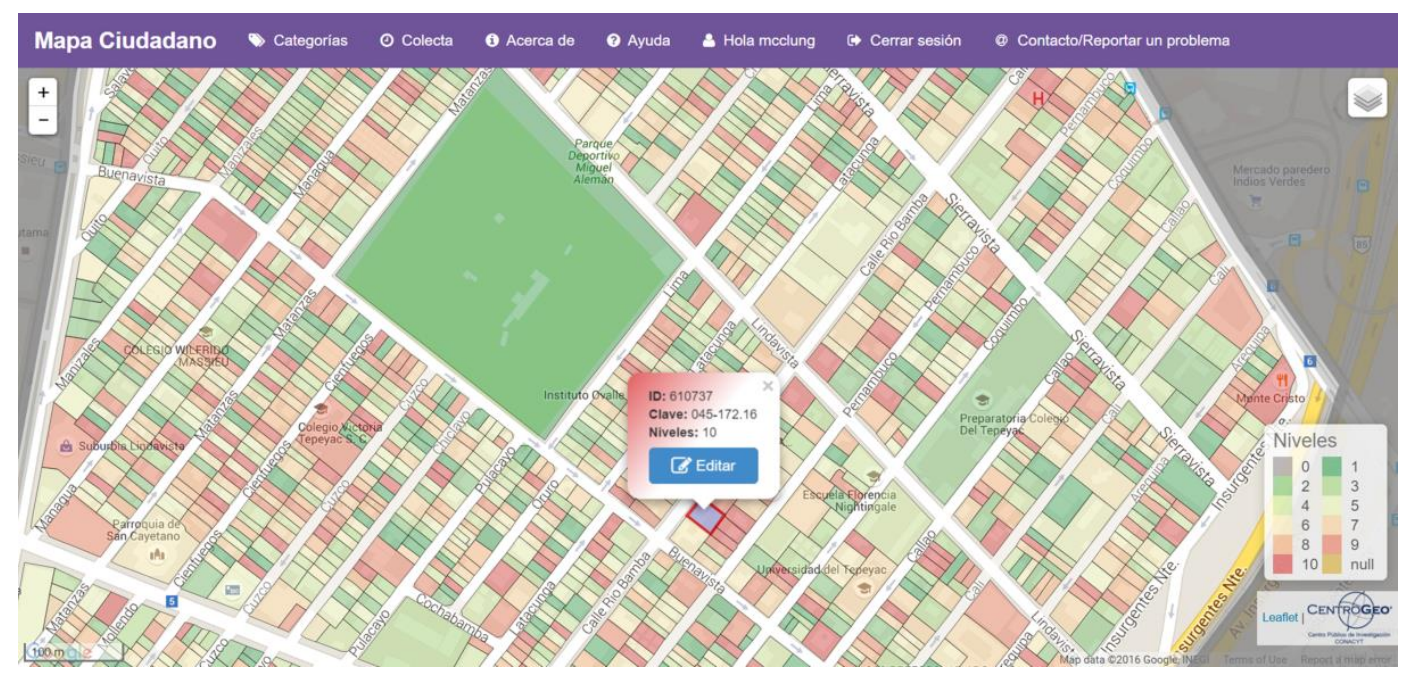

Figure 2: Parcel lot data collection for Lindavista 


\section{Discussion and future work}

In the case study presented here, the nature of what local people need drives the way the platform is developed. Exposing the locations of situations that they perceive as requiring attention and the need to collect data that is not readily available from the authorities provide the foundation for the design of an online digital platform that aids them to do both. The platform incorporates the possibility of including official data for citizens to validate, edit or delete. It also offers a voting strategy to obtain an empirical measure of how citizens perceive other contributions in the study area. Additionally, it provides a mechanism to crowdsource information with respect to the levels of construction in each parcel lot in the neighbourhood. The identification of issues that, potentially at least, allowfor negotiation between citizens and their local authorities helps the platform play an important role as a management tool in the citizen-government process.

By collecting geospatial data, citizens are empowered because, together, they can get a better overall picture of the neighbourhood and can put forward issues that may not necessarily be on the government's agenda. Also, properly conveyed and well communicated, these matters can open the door for better negotiation with their local authorities to help improve their quality of life.

Although this ongoing study focuses on a few variables for a specific neighbourhood, many other neighbourhoods in the metropolitan area share their situation and experience similar ailments. Once this exercise reaches a more mature state, efforts will be directed towards the appropriateness and possibility of deployment in other neighbourhoods, or even at municipal level. A relevant contribution is the use of crowdsourced parcel lot data which can be compared with the official land use map.

This paper does not delve into the issue of the standardization of citizen-generated information. However, it is worth looking into the contributions of the crisis management research community on this topic, a community which uses the Open Geospatial Consortium's (OGC) Sensor Web Enablement (SWE) and the Observation and Measurements (O\&M) schema (Kamel Boulos et al., 2011; Schade et al., 2013).

In terms of platform enhancements, it is important to review the current data model and structure to allow users to edit their own observations. Although this has not yet been implemented, there have been few incidences requiring correction. The system could also profit from an approach that involves measuring the level of (dis)agreement among several users in relation to a particular observation filed by another user; this information could be used to compute a degree of trustworthiness for subsequent observations submitted by the same user, similar to building a reputation score for users. The way geometries are handled could also be improved because currently, when the front-end is loaded or the information from the database is updated, all the geometries are read and a single feature collection is sent to the browser. This is not efficient when dealing with nearly four thousand parcel lots. While it would be possible to create different feature collections for each city block, it would also be inefficient to add hundreds of layers to the Leaflet map. Further research and development is needed for this portion of the platform to be optimized in order to be able to 
load thousands of parcel lots. A broader, more general incarnation of the platform, containing several thousand parcel lots more, could be used at the municipal level.

An interesting preliminary result concerns how citizens are accessing the platform. Even though it was built to be responsive on mobile devices and desktop environments, initial results show that $84 \%$ of the users were working on desktop computers, $10 \%$ on mobile phones and $6 \%$ on tablets. Further research is needed in terms of platform appropriation and technology: might it be possible, for example, to reach more citizens if there was an existing native mobile app? Another interesting topic to look into is the possibility of integrating these citizen involvement mechanisms into existing social networks that users are already familiar with.

The inclusion of official data sources for users to validate is an improvement over other ways to crowdsource data. The map is already populated at the start of the project and citizens do not have to build the database from scratch. This was well received by citizens. However, because of the dynamics of the neighbourhood, an up-to-date database of current businesses is very difficult to achieve. This approach, then, provides a faster way to update official records, spending less effort on fieldwork.

The voting strategy implemented as a first approach to assessing the credibility of VGI seems to be useful. It is in the longer term, however, that the true value of this approach will be seen: once there are enough citizen observations and votes per observation to allow for empirical distributions of users to be built and observations to be interpreted. A subsequent iteration of the platform will probably incorporate the power of visual analytics, in order to display meaningful visualizations and so help us understand and make better sense of data generated by citizen observations and votes.

Initial results from the parcel lots exercise indicate that not all users, when standing at street level, perceive levels of construction in the same way, although initially it had been thought that it would be straightforward to allocate a number with respect to the level of construction in a given lot. As a result, values of levels of construction for parcel lots were being overwritten. If the proposed voting strategy is successful, it could be possible to develop and incorporate something similar for the parcel lot map. Careful consideration will follow after enough data has been gathered, and the value and usefulness of the voting strategy are assessed. Additionally, an extended data model for the parcel lots might be put in place in order to keep track of all the modifications with regard to the levels of construction that users are making, and to try to shed some light on citizens' perceptions when collecting this type of data.

Lastly, it will be interesting to try and incorporate a way of updating reported observations when their status changes - for instance, when a pothole is fixed or an establishment goes out of business. This would give citizens, and perhaps the authorities, a way to update their records, keeping the map alive, not only with observations and complaints but also with changes and enhancements seen and perceived by the local people themselves. 


\section{References}

Atzmanstorfer, K., Resl, R., Eitzinger, A., \& Izurieta, X. (2014). The GeoCitizen-approach: community-based spatial planning - an Ecuadorian case study. Cartography and Geographic Information Science, 41(3), 1-12. http:/ / doi.org/10.1080/15230406.2014.890546

California Academy of Sciences (2015). iNaturalist. Retrieved 9 November 2015, from http://www.inaturalist.org/

cartoDB. (2015). cartoDB. Retrieved 9 November 2015, from https://cartodb.com/

Fonte, C. C., Bastin, L., See, L., Foody, G., \& Estima, J. (2015). Good practice guidelines for assessing VGI data quality. In The 18th AGILE International Conference on Geographic Information Science.

Goodchild, M. F. (2007). Citizens as sensors: The world of volunteered geography. GeoJournal, 69(4), 211-221. http://doi.org/10.1007/s10708-007-9111-y

Haklay, M. (2013). Citizen Science and Volunteered Geographic Information: Overview and Typology of Participation. In D. Sui, E. Sarah \& M. Goodchild (Eds.), Crowdsourcing Geographic Knowledge: Volunteered Geographic Information (VGI) in Theory and Practice. Berlin: Springer (pp. 105-122). http://doi.org/10.1007/978-94-007-4587-2_7

Heipke, C. (2010). Crowdsourcing geospatial data. ISPRS Journal of Photogrammetry and Remote Sensing, 65(6), 550-557. http://doi.org/10.1016/j.isprsjprs.2010.06.005

Imperial College London (2015). EpiCollect+. Retrieved 9 November 2015, from http://www.epicollect.net/

INEGI (Instituto Nacional de Estadística y Geografía) (2015). Directorio Estadístico Nacional de Unidades Económicas. Retrieved 10 September 2015, from http://www.inegi.org.mx/est/contenidos/proyectos/denue/presentacion.aspx

Johnson, P. A. \& Sieber, R. E. (2013). Situating the adoption of VGI by government. In D. Sui, E. Sarah, \& M. Goodchild (Eds.), Crowdsourcing Geographic Knowledge: Volunteered Geographic Information (VGI) in Theory and Practice. Berlin: Springer (pp. 65-81). http://doi.org/10.1007/978-94-007-4587-2

Jovchelovitch, S. \& Priego-Hernandez, J. (2015). Bottom-up social development in favelas of Rio de Janeiro: a toolkit. Retrieved 10 September 2015, from http://eprints.lse.ac.uk/62563/1/ToolkitSocialDevelopmentLSE2015.pdf

Kamel Boulos, M. N., Resch, B., Crowley, D. N. and others, (2011). Crowdsourcing, citizen sensing and sensor web technologies for public and environmental health surveillance and crisis management: trends, OGC standards and application examples. International Journal of Health Geographics, 10(1), 67. http://doi.org/10.1186/1476-072X-10-67

mySociety (2012). Fix My Street. Retrieved 14 March 2016, from https://www.fixmystreet.com/

OpenTreeMap (2015). OpenTreeMap. Retrieved 9 November 2015, from https://www.opentreemap.org/

Schade, S., Díaz, L., Ostermann, and others. (2013). Citizen-based sensing of crisis events: sensor web enablement for volunteered geographic information. Applied Geomatics, 5(1), 3-18. http://doi.org/10.1007/s12518-011-0056-y .

University College London (2015). GeoKey. Retrieved 9 November 2015, from http://geokey.org.uk/

Ushahidi (2015). Ushahidi. Retrieved 9 September 2015, from https://www.ushahidi.com/ 